\title{
Comparison of Ion Internal Transport Barrier Formation between Hydrogen and Helium Dominated Plasmas*)
}

\author{
Kenichi NAGAOKA ${ }^{1,2)}$, Hiromi TAKAHASHI ${ }^{1,2)}$, Kenji TANAKA $^{1)}$, Masaki OSAKABE ${ }^{1,2)}$, \\ Sadayoshi MURAKAMI ${ }^{3)}$, Shogo MAETA ${ }^{3)}$, Masayuki YOKOYAMA ${ }^{1)}$, Keisuke FUJII ${ }^{4)}$, \\ Haruhisa NAKANO ${ }^{1,2)}$, Hiroshi YAMADA ${ }^{1,2)}$, Yasuhiko TAKEIRI ${ }^{1,2)}$, Katsumi IDA ${ }^{1,2)}$, \\ Mikiro YOSHINUMA ${ }^{1,2)}$ and the LHD Experiment Group \\ ${ }^{1)}$ National Institute for Fusion Science, Toki 509-5292, Japan \\ ${ }^{2}$ SOKENDAI (The Graduate University for Advanced Studies), Toki 509-5292, Japan \\ ${ }^{3)}$ Department of Nuclear Engineering, Kyoto University, Kyoto 606-8501, Japan \\ ${ }^{4)}$ Department of Mechanical Engineering and Science, Kyoto University, Kyoto 615-8540, Japan
}

(Received 9 December 2015 / Accepted 22 June 2016)

\begin{abstract}
Ion internal transport barrier (ITB) was formed in both hydrogen discharge and helium dominated discharge. The central ion temperature was investigated as a function of the hydrogen density ratio $\left(n_{\mathrm{H}} /\left(n_{\mathrm{H}}+n_{\mathrm{He}}\right)\right)$. The central ion temperature increases with the decrease of the hydrogen density ratio, while the electron temperature does not change significantly. The experimentally observed ion temperature was not reproduced by the prediction of TASK-3D modeling. The dominant activity in the density fluctuation measured by the phase contrast imaging is consistent with ion temperature gradient mode and they are almost identical between the hydrogen dominated and helium dominated plasmas.
\end{abstract}

(C) 2016 The Japan Society of Plasma Science and Nuclear Fusion Research

Keywords: ion ITB, helical plasma, isotope effect, deuterium experiment

DOI: $10.1585 / \mathrm{pfr} .11 .2402106$

\section{Introduction}

The deuterium experiment is planned in the Large Helical Device (LHD) project. The physics mechanism of the isotope effect is one of the most important subjects in the deuterium experiment [1], where the isotope effect means the differences of confinement characteristics between a hydrogen discharge and a deuterium discharge.

In tokamak plasmas, it is widely recognized that the heating power threshold of the L-mode to H-mode transition depends on the ion species. In the deuterium discharges in a tokamak, the power threshold is smaller than that in the hydrogen discharges $[2,3]$. The transport improvement in the core region was also identified in JT$60 \mathrm{U}$ [3]. However, the physics mechanism is not yet understood.

A gyrokinetic simulation of the ion temperature gradient (ITG) mode in the LHD configuration shows the reduction of zonal flow damping rate in a deuterium plasma. As a result, the enhancement of zonal flow and the suppression of ion heat transport are predicted in the deuterium discharges in LHD [4].

The transport characteristics and their ion species dependence have been investigated using hydrogen and helium dominated plasmas in LHD. In this paper, the depen-

author's e-mail: nagaoka@nifs.ac.jp

*) This article is based on the presentation at the 25th International Toki Conference (ITC25). dence of the heat transport characteristics of ion internal transport barrier (ion ITB) discharges upon the density ratio of hydrogen to helium is presented. In the next section, the ion ITB plasma and the experimental observations are presented. The density fluctuation characteristics are also discussed. Finally, important points for research on isotope effect in deuterium experiment in LHD are discussed.

\section{Ion ITB Discharges}

The experimental stage is the Large Helical Device (LHD) at the National Institute for Fusion Science (NIFS) [5]. The coil systems are composed of super conductors. The magnetic field strength is up to 3T. The major and the averaged mirror radii are $3.75 \mathrm{~m}$ and $0.6 \mathrm{~m}$, respectively. The toroidal and poloidal mode numbers are $n=10$ and $m$ $=2$, respectively.

In order to replace the gas species, intense discharge cleaning was carried out before the experiment. The long pulse helium discharges with the duration time of 60 sec were repeated with ion cyclotron range of frequency (ICRF) heating [6-8]. The hydrogen recycling was reduced significantly.

Next the ion ITB discharge with helium gas puffing was performed with neutral beam injection (NBI) heated plasmas. The hydrogen ion ratio $\left(n_{\mathrm{H}} /\left(n_{\mathrm{H}}+n_{\mathrm{He}}\right)\right)$ was scanned from 0.34 to 0.78 on shot by shot basis by changing the gas puff from helium to hydrogen. Figure 1 shows 

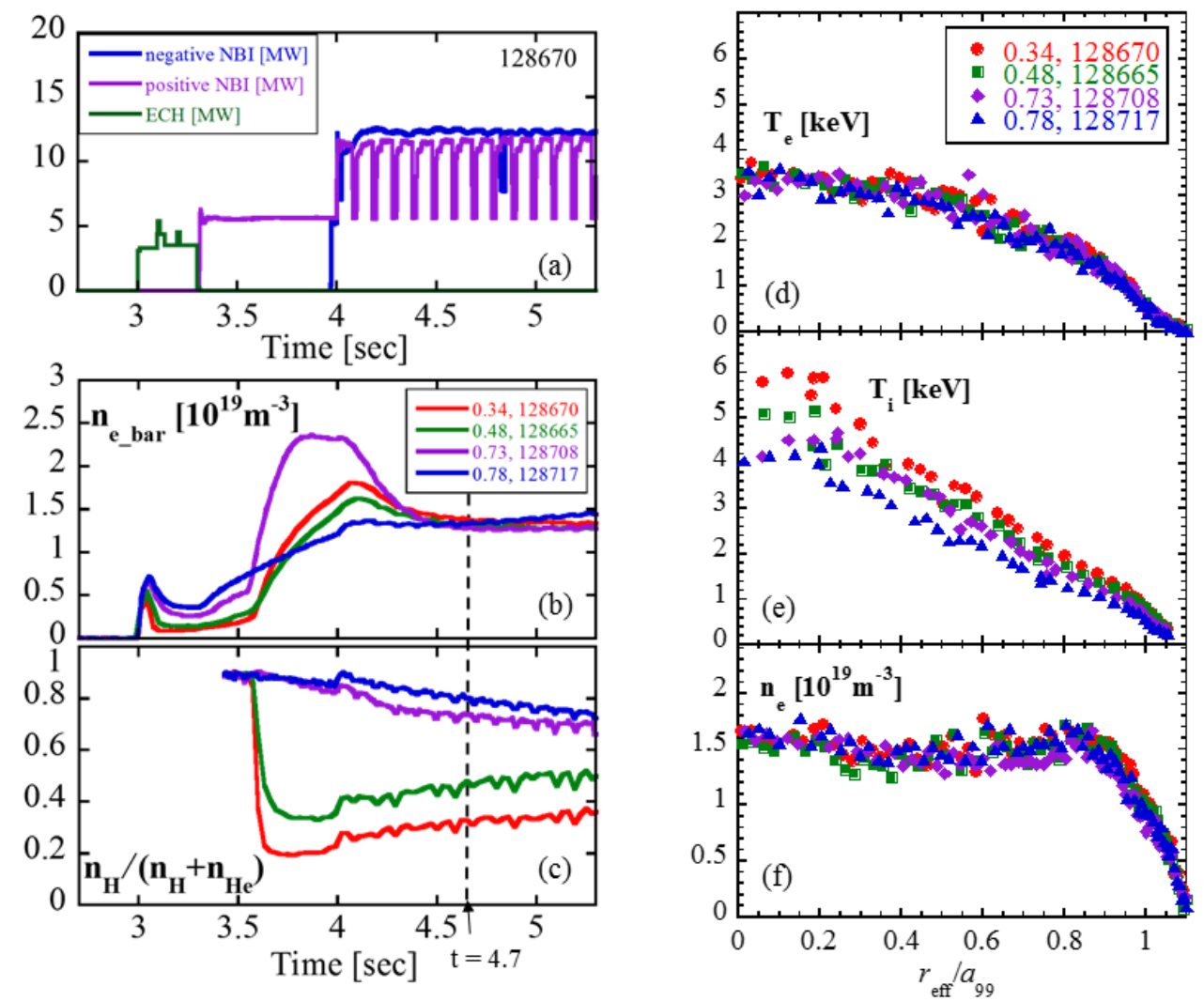

Fig. 1 (a) Time evolution of heating power of negative NBI, positive NBI and ECH. The port-through powers of negative-ion-based NBIs (tangential injection) and the positive-ion-based NBIs (perpendicular injection) are 10.1-12.3 MW and 11.6-11.7 MW, respectively. (b) Time evolutions of the line averaged density and (c) hydrogen ratio $\left(n_{\mathrm{H}} /\left(n_{\mathrm{H}}+n_{\mathrm{He}}\right)\right)$ of four shots. The helium gas was puffed at $t=3.5-3.55$ and 3.6-3.75 sec for 128670, and $t=3.5-3.54$ and 3.6-3.75 sec for 128665 . The hydrogen gas was puffed at $t=3.5-3.9 \mathrm{sec}$ for 128708. No gas puff was operated for 128717. Radial profiles of (d) electron temperature, (e) ion temperature and (d) electron density of four shots with the time of $t=4.7 \mathrm{sec}$.

four discharges and we compare plasmas with the same line averaged density of $1.3 \times 10^{19} \mathrm{~m}^{-3}$ and with different hydrogen ion ratio. Figures $1(\mathrm{~d}-\mathrm{f})$ show the typical profiles of electron temperature, ion temperature, and electron density. The one perpendicular NBI (BL4) was modulated with $\mathrm{ON} / \mathrm{OFF}=80 \mathrm{msec} / 20 \mathrm{msec}$ for the calibration of charge-exchange spectroscopy (CXS) diagnostic and the ion temperatures are estimated by the line spectrum of carbon impurities [9]. The hydrogen ratio was experimentally evaluated by line emissions of hydrogen and helium [10]. The total hydrogen beam current injected into the plasma is about 350A, and the particle fueling rate for the hydrogen beam is the order of $10^{21} \mathrm{~s}^{-1}$. The minimum hydrogen ratio $\left(n_{\mathrm{H}} /\left(n_{\mathrm{H}}+n_{\mathrm{He}}\right)\right)$ with helium gas puffing was 0.34 in the condition of low hydrogen recycling wall. The wall condition with very low hydrogen recycling was sustained in only a few discharges and the wall condition changes shot by shot during the experiment. The electron density near the edge $\left(r_{\text {eff }} / a_{99}>0.7\right)$ slightly changes depending on the wall condition and gas puff condition, however the electron density in the core $\left(r_{\text {eff }} / a_{99}<0.5\right)$ is almost identical among the four shots compared in Fig. 1. Therefore, the clear change of particle transport was not identified in the experiment.
The electron temperature profiles are identical among the four plasmas presented in Fig. 1. On the other hand, the central ion temperature of around $6 \mathrm{keV}$ was observed with hydrogen ion ratio of 0.34 , and the central ion temperature decreased with the increase of hydrogen content. In order to evaluate the heat transport in the ion ITB discharges, the experimental observation was compared with the prediction with the empirical transport model. The thermal diffusivity in the transport model is given by

$$
\chi_{\mathrm{i}, \mathrm{e}}=\chi_{\mathrm{i}, \mathrm{e}}^{\mathrm{Neo}}+\chi_{\mathrm{i}, \mathrm{e}}^{\mathrm{TB}},
$$

where the subscript "Neo" and "TB" indicate the neoclassical transport and turbulent transport components, respectively. The turbulent diffusivity is modeled based on gyroBohm scaling with temperature gradient effect,

$$
\chi_{\mathrm{i}}^{\mathrm{TB}}=\chi_{\mathrm{iGB}} \cdot\left(\frac{a}{T} \frac{\partial T}{\partial \rho}\right),
$$

where $\chi_{\mathrm{iGB}}, a, T, \rho$ are gyroBohm thermal diffusivity, minor radius, temperature and normalized minor radius, respectively [1]. Figure 2 shows the comparison of experimental observations and the model prediction calculated with TASK-3D transport code $[12,13]$. In the model calculation, the population of hydrogen ions, helium ions and 
carbon impurity ions are taken into account, and the transport coefficients are assumed to be the same among different ion species. The model prediction in the hydrogen ion

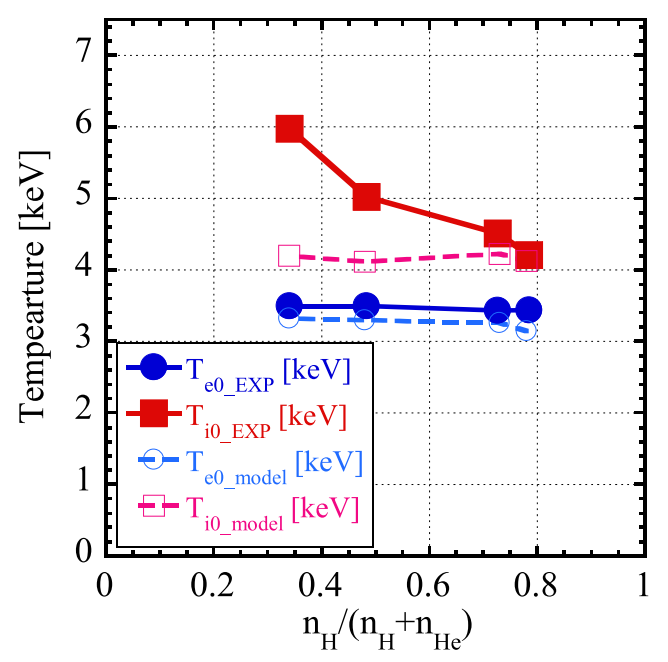

Fig. 2 Ion and electron temperature at the magnetic axis $\left(r_{\text {eff }} \sim\right.$ $0)$ as a function of hydrogen ion ratio $\left(n_{\mathrm{H}} /\left(n_{\mathrm{H}}+n_{\mathrm{He}}\right)\right)$. Solid lines show the experimental observation, and the dashed lines show the prediction of the empirical model. dominated regime is consistent with the experimental observation. However, the ion temperature in the helium ion dominated regime is higher than the model prediction. In order to evaluate the NBI heating power more accurately, GNET code was utilized. The full orbits of fast ions during the energy slow-down are calculated with multi-ion bulk plasma in GNET [14], while an analytical formula of Fokker-Plank equation with prompt finite orbit effect is utilized in TASK-3D code [13]. The total ion particle number changes with the hydrogen ratio, however the ion heating power normalized by the particle number evaluated with GNET code is almost identical and is $(0.14 \pm 0.01)$ $\times 10^{-19} \mathrm{MW} /$ particle for four cases due to the decrease of critical energy $\left(E_{\mathrm{cr}} \sim(Z / A)^{2 / 3}\right)$ for helium dominated plasmas. Therefore it was identified that the ion heat transport changes with the ion species. On the other hand, the electron temperature does not depend on the ion species content and agrees with the model prediction. Theoretical research for developing predicable model with transport improvement with $Z_{\text {eff }}$ or $A_{\text {eff }}$ dependence is in progress [15], which is beyond the scope of this paper.

In order to discuss anomalous transport, the density fluctuation properties measured with the phase contract imaging (PCI) diagnostic [16] were compared between hy-

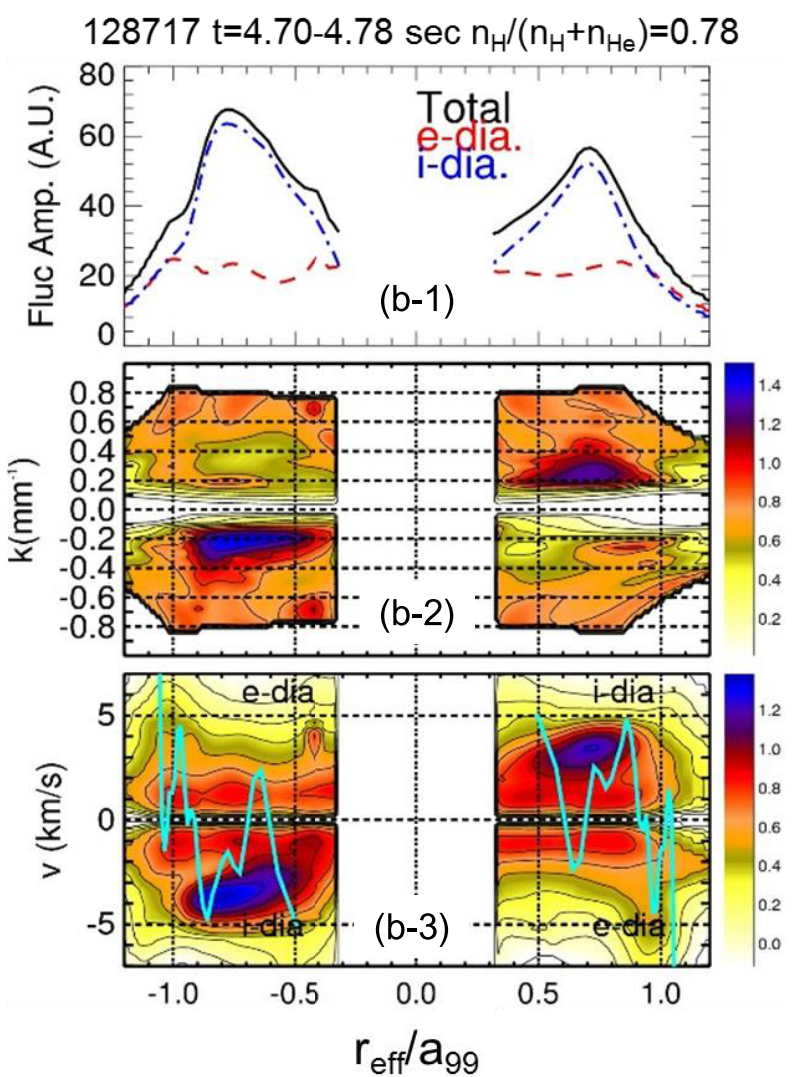

Fig. 3 Radial profiles of the density fluctuation measured with PCI. (Upper: a/b-1) Amplitudes of density fluctuation directed electron diamagnetic drift, ion diamagnetic drift and total of them, (Middle: a/b-2) the distribution of the perpendicular wave number dependence, and (Bottom: a/b-3) the distribution of the perpendicular velocity to the magnetic field in the laboratory frame. The density fluctuation with frequency range of $20-500 \mathrm{kHz}$ are plotted. The color contour represents the intensity in the logarithmic scale. The profiles of poloidal rotation velocity evaluated by the CXS are also plotted in the $(\mathrm{a} / \mathrm{b}-3)$. 
drogen ion dominated and helium ion dominated plasmas. Figure 3 shows radial profiles of (upper) the amplitude of the density fluctuation, (middle) intensity of the wave number of the density fluctuation, and (bottom) intensity of phase velocity of the density fluctuation in the laboratory frame. The density fluctuation with a frequency of $20-500 \mathrm{kHz}$ are analyzed and plotted in Fig. 3. The density fluctuation rotating in the direction of ion-diamagnetic drift with the wave number of $0.1-0.4 \mathrm{~mm}^{-1}$ was observed in the both plasmas with hydrogen ratio of 0.34 and 0.78 . Although the mode identification is difficult from only the PCI observation, ion temperature gradient (ITG) mode was identified to be dominant mode in the high ion temperature discharges in LHD from the comparison between the experimental observation and gyrokinetic calculation [17-19]. The dominant mode observed in this experiment is very similar to that obtained in the previous experiment, thus the mode seems to be the ITG mode. The global characteristics of the density fluctuation observed with PCI, such as, the amplitude profile, peak level, wave number profile, rotation, etc. are almost identical between the two plasmas, while the ion heat transport is different between them. The observed wave number spectra seem to be reasonable, because the characteristics scale of ITG is determined by the ion Larmor radius $\left(\rho_{\mathrm{i}} \sim\left(A^{1 / 2} / Z\right)\right.$, where $A$ and $Z$ are mass number and atomic number, respectively), which is identical between hydrogen plasma $(A=1$ and $Z=1)$ and helium plasma $(A=4$ and $Z=2)$. However, the observation of fluctuation amplitude seems to be inconsistent with the fact that the ion heat transport is different between helium dominated and hydrogen dominated plasmas. Based on the mixing length theory, the thermal diffusivity is given by $\chi_{i} \sim \gamma / k^{2}$, where $\gamma$ and $k$ are the growth rate and wave number perpendicular to the magnetic field, and the fluctuation measurements suggest that the growth rate changes depending on ion species. In other words, the phase relation between two fluctuating quantities such as density and temperature changes and the induced turbulent heat flux changes as well.

\section{Discussion and Summary}

The transport of the plasma with ion ITB is compared between hydrogen dominated plasma and helium dominated plasma. The experimental observation clearly shows that the ion temperature depends on the density ratio of hydrogen ions, and the ion temperature is higher in the low hydrogen density ratio. On the other hand, no difference in particle transport and electron heat transport were identified in the present experiment. The comparison with the developed empirical transport model based on the gyroBohm scaling with temperature gradient factor indicates the improvement depending on the hydrogen ion ratio in the ion heat transport. The density fluctuation measured with PCI indicates that the density fluctuation rotating in the ion-diamagnetic drift direction is dominated in the plasmas and the global characteristics of the mode do not change depending on the ion species. The importance of evaluation of growth rate is highlighted, while the physics mechanism of the ion transport improvement is a still open issue.

The similar behavior was observed when small amount of impurity is injected into the plasma, such as carbon pellet injection, nitrogen gas puffing, etc. In the case of carbon pellet injection, the ion heat transport is clearly reduced with the carbon density of $1 \%$ of bulk ion density and effective charge number $Z_{\mathrm{eff}}$ of around 2 . The detailed transport analysis shows that the ion thermal diffusivity depends on the carbon density in the plasma [20]. These experimental results indicate the need to investigate the dependence of ion thermal diffusivity on $Z_{\text {eff }}$ and/or $A_{\text {eff }}$. The further upgrade of the analytical tool TASK-3D code is ongoing [21], and the theoretical modeling, which includes $Z_{\text {eff }}$ and/or $A_{\text {eff }}$ dependence for heat transport improvement, is discussed in another paper [15].

In this paper, the transport characteristics of different ion species were experimentally compared in the condition that the ion Larmor radii were kept a constant, which is considered as an important reference experiment for studying the physics mechanism of isotope effect, which is planned in coming deuterium plasma experiment in LHD.

\section{Acknowledgements}

The authors thank Profs. H. Sugama, M. Nunami, M. Nakata for fruitful discussions and LHD project members for their contribution to the operation of LHD. This research was supported by NIFS13KLPR017 and NIFS14KKGR003.

[1] M. Osakabe et al., the 25th International Toki Conference, invited talk (I-1), 3rd Nov. 2015, Toki, Japan.

[2] For example, Y.R. Martin et al., J. Phys.: Conf. Ser. 123, 012033 (2008).

[3] H. Urano et al., Nucl. Fusion 52, 114021 (2012).

[4] T.H. Watanabe, H. Sugama and M. Nunami, Nucl. Fusion 51, 123003 (2011).

[5] O. Motojima et al., Nucl. Fusion 43, 1674 (2003).

[6] H. Takahashi, M. Osakabe et al., Plasma Fusion Res. 9, 1402050 (2014).

[7] H. Takahashi, M. Osakabe, K. Nagaoka et al., J. Nucl. Mater. 463, 1100 (2015).

[8] K. Nagaoka, H. Takahashi, S. Murakami et al., Nucl. Fusion 55, 113020 (2015).

[9] M. Yoshinuma et al., Fusion Sci. Technol. 58, 375 (2010).

[10] M. Goto, S. Morita, K. Sawada et al., Phys. Plasmas 10, 1402 (2003).

[11] S. Murakami et al., the 26th IAEA Fusion Energy Conf. (St Petersburg, 13 - 18 October 2014) TH/P6-38.

[12] S. Murakami et al., 20th International Stellarator- Heliotron Workshop (ISHW), October 5 - 9, 2015, Greifswald, Germany, P1S2-23.

[13] S. Murakami et al., Plasma Phys. Control. Fusion 57, 054009 (2015).

[14] S. Murakami et al., Nucl. Fusion 46, S425 (2006). 
[15] S. Maeta et al., the 25th international Toki conference, 2015, Toki, Japan. P1-116.

[16] K. Tanaka et al., Rev. Sci. Instrum. 79, 10E702 (2008).

[17] K. Tanaka et al., Plasma Fusion Res. 5, S2053 (2010).

[18] M. Nunami et al., Plasma Fusion Res. 6, 1403001 (2011).
[19] M. Nunami et al., Phys. Plasmas 19, 042504 (2012).

[20] K. Nagaoka et al., Nucl. Fusion 51, 083022 (2011).

[21] M. Yokoyama et al., 20th International Stellarator- Heliotron Workshop (ISHW), October 5 - 9, 2015, Greifswald, Germany, P2S3-49. 